JUN 121987

BROOKHAVEN NATIONAL LABORATORY ON- $8100914.7--4$

February 1987

BNL-39764

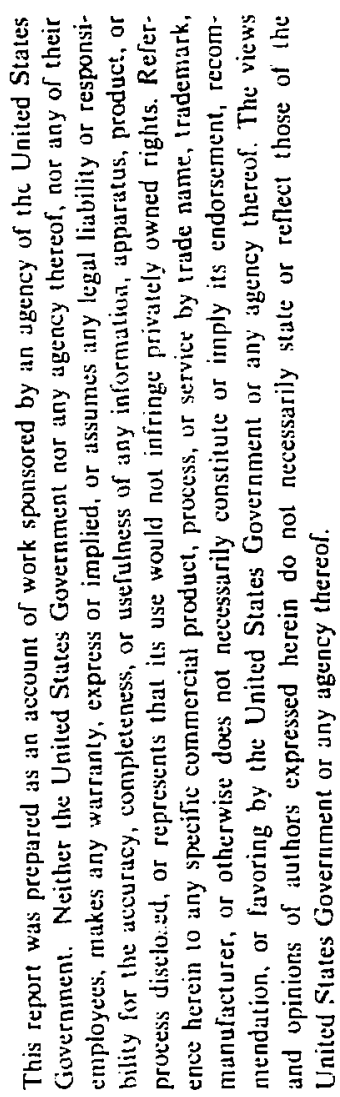

\title{
TOWARD A PSEUDOFERMION CALCULATION \\ OF THE HADRONIC MASS SPECTRUM
}

\author{
M. Campostrini
}

INFN Sezione di Pisa, Italy and BNL

BNL- -39764

K.J.M. Moriarty

DE87 010441

Dalhousie University, Halifax, Canada

\section{J. Potvin ${ }^{*}$}

Physics- Đepartment, Brookhaven National Laboratory

Uptor, NY 11973

\section{Claudio Rebbi}

Physics Department

BNL, Upton, NY, and Boston University, Boston, MA

$\mathrm{QCD}$, on a $10^{3} \cdot 32$ lattice at $\beta=5.7$.The fermions are of the KogutSusskind type ( 3 flavors) with mass $m a=0.05$. It is found that the hadron propagators take more than 10000 Metropolis iterations to equilibrate, a factor 5 more than what is needed in a typical quenched calculation. We present also our results on the effects of dynamical quarks on the Wilson loops.

- Talk given by J. Potvin at Lattice Gauge Theory '86, BNL, 14-19 September 1986, to be published in the Proceedings.

This manuscript has been anthored under contract number DE-AC02-76CH00016 with the U.S. Department of Energy. According1y, the U.S. Government retains a non-exclnsive, royalty-free license to publish or reproduce the published form of this contribation, or allow others to do so, for U.S. Government purposes. 
TOWARD A PSEUDOFERMION CALCULATION OF THE HADRONIC MASS SPECTRUM

\section{J. POTVIN}

Physics Department, Brookhaven National Laboratory

Upton, NY 11973, USA

in collaboration with:

M. CAMPOSTRINI, INi N, Pisa, Italy

K.J.M. MORIARTY, Dalhousi University, Halifax, Canada

C. REBBI, BNL, Upton, NY anł Boston Univ., Boston, MA

\section{ABSTRACT}

We present our preliminary results of a pseudofermion simulation of $\mathrm{QCD}$, on a $10^{3} \cdot 32$ lattice at $\beta=5.7$. The iermions are of the Kogut-Susskind type ( 3 fiavors) with mass $m a=0.05$. It is found that the badron propagators take more inan 10000 Metropolis iterations to equilibrate, a factor 5 more than what is needed in a typical quenched calculation. We present also our results on the effects of dynamical quarks on the Wilson loops.

\section{MOTIVATIONS}

The calculation of the hadron mass spectrum without including the dynamicai quarks (i.e. the quenched approximation) has lead to very encouraging results. For one, the caiculated hadron masses have been found consistent with the experimental values, within statistical and systematic error. Also, the breaking of chiral symmetry and the Goldstone nature of the pion have been seen. ${ }^{1}$ 
Of course,the quenched approximation is not the whole srory. since QCD is a theory about giuons AND guarks. It is therefore important to verify that, upon the re-introduction of the dynamical quarks into the picture, the above results will not change much. Moreover, dynamical quark efiects should be visible in the heavy quark potential, at separations beyond one Fermi where hadronization takes place.

It has been known for a long time that the unguenched simulation of QCD is much more demanding on computer resources. It is only with the receni availability of supercomputer time that algorithms with dynamical fermions have for the first time been tried out and tested on reasonably large lattices $\left(\geq 8^{4}\right)$ and couplings $(\geq 5.0)$, and with small masses $(\leq 0.10)$. Exampies of such algorithms are presented and discussed in this Conference Proceedings.

Most physics applications of these algoritinms have been concerned with $\mathrm{QCD}$ at finite temperature. In contrast, only three large scale studies of the:hadron-mass spectrum have been carried out:

1. a Langevin calculation by Fukugita et al. ${ }^{2}$ on a $9^{3} \cdot 18$ lattice, at $\beta=5.5$ with Wilson fermions. For more details, see Fukugita's talk in these Proceedings.

2. a pseudofermion simulation by Fucito et al., ${ }^{3}$ on a $12^{3} \cdot 24$ lattice at $\beta=5.4$ with three flavors of Kogut-Susskind fermions. Three mass values were tried: $0.02,0.05$, and 0.10 . Their statistics was limited however, with only nine propagators per mass.

3. another pseudofermion calculation, by Laerman et al., ${ }^{4}$, this time with the group $S U(2)$. The lattice size was $8^{3} \cdot 16$ and $1.85 \leq \beta \leq 2.50$; four flavors of Kogut-Susskind fermions were used in the mass range $.035 \leq m a \leq 0.200$.

We have continued this exploratory effort with another pseudofermion calculation, at mass $m a=0.05$ with three flavors of Kogut-Susskind quarks. The lattice size was $10^{3} \cdot 32$, at $\beta=5.7$. In this talk I will present our preliminary results with emphasis on the equilibrium time required for the Wiison loops $(W(1,1))$ and pion propagator $\left(P_{t t_{0}}\right)$. Our conclusions will be that it is very difierent for both quantities: thousands more iterations (i.e. link upgrades) are needed for $W(1,1)$ than for $P_{t_{0}}$; this is also many more than what is required in a calculation of $P_{t_{0}}$ in the quenched approximation.

Our simulation was carried out on a CDC CYBER 205 based at Rockville Md., thanks to the generosity of the Control Data Corporation. 


\section{THE ALGORITHM}

According to the procedure described in Ref. 5 and Ref. 6, the pseudofermions $\phi_{z}$ are upgraded first with a heat bath algorithm in the background of fixed gauge fields. Here the $\phi_{x}$ are modified in the following way,

$$
\begin{aligned}
\phi_{z} I & =\phi_{x}+\delta \phi_{z} \\
\delta \phi_{z} & =\frac{1}{\sqrt{2+m^{2}}} V_{x}+\frac{1}{2+m^{2}}\left(D^{\dagger}+m\right)(D+m) \phi_{x}
\end{aligned}
$$

$V_{x}$ being a Gaussian random number of unit variance and $(D+m)$ the Dirac operator. Such an upgrade is done 300 times, calculating and accumulating the reaction $J_{x}^{\mu}$ every 12 sweeps. $J_{x}^{\mu}$ is needed in the calculation of the variation of the total action Ref. 6:

$$
\delta S=\delta S_{\text {Gauge }}-\frac{1}{8} n_{f} \sum_{x, \mu}\left(\operatorname{Tr}\left\langle J_{x}^{\mu}\right\rangle \delta U_{x}^{\mu}+\text { H.C. }\right)
$$

$\left\langle J_{z}^{\mu}\right\rangle$ is thus evaluated from a sample of 25 values, in similarity with the calculations of Ref. 3 and Ref. 7. A distinct feature of our study is that not all $\phi_{x}$ are upgraded at each pseudoferimion sweep but only a subset of approximately 1000, chosen in a uniformly random fashion (the sites being still distanced at least 3 steps away to allow for vectorization). This procedure still satisfies detailed balance, and eliminates any potential directional correlations due to upgrading. With the use of asynchronous $I / O$, it also shortens the pseudofermion upgrade while maintaining a reasonable vector length.

After the 300 pseudofermion sweeps,the link variables are then upgraded with a standard Metropolis procedure, at a rate of 8 upgrades per lint. Here the acceptance is $81 \%$. This coupled heat bath/Metropolis update (or "iteration") took 0.3 mililiseconds per link and was run for a total of 9000 iterations (14500 after this Meeting). The Wilson loops were measured every 10 iterations and propagators every 500.

\section{WILSON LOOPS}

Figures 1 and 2 show the evolution of the $1 \times 1,2 \times 2,3 \times 3$ and $4 \times 4$ Wilson loops with respect to iteration number. Clearly, the smaller loops have equilibriated after $\mathbf{2 5 0 0}$ iterations, more or less. The larger ones are of course slower and show equilibration after 4000 iterations.

After 14500 iterations, we get

$$
W(1,1)=0.5816 \pm .0002
$$



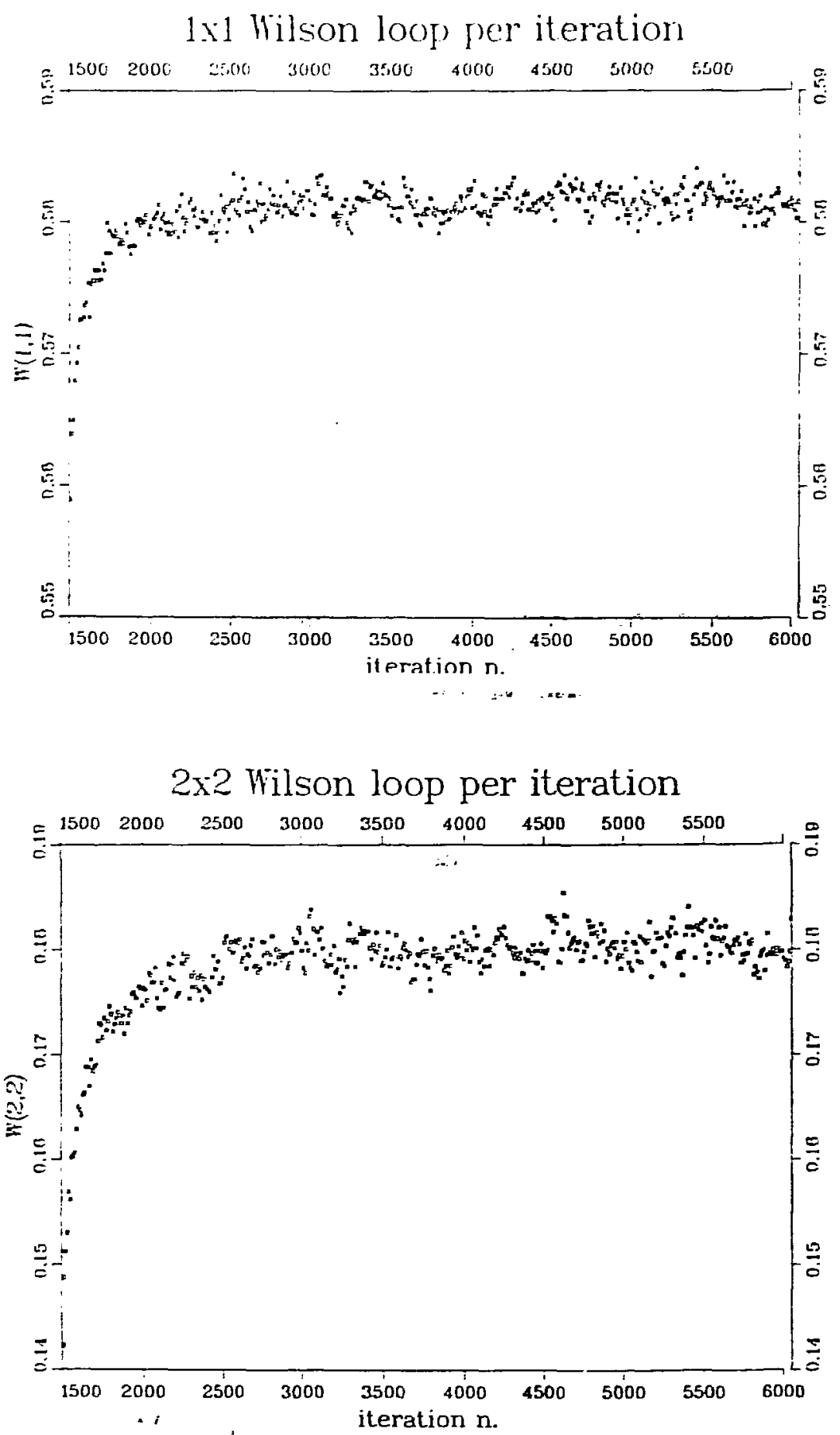

Fig. 1 : $1 \times 1$ and $2 \times 2$ Wilson loops versus the iteration number. 

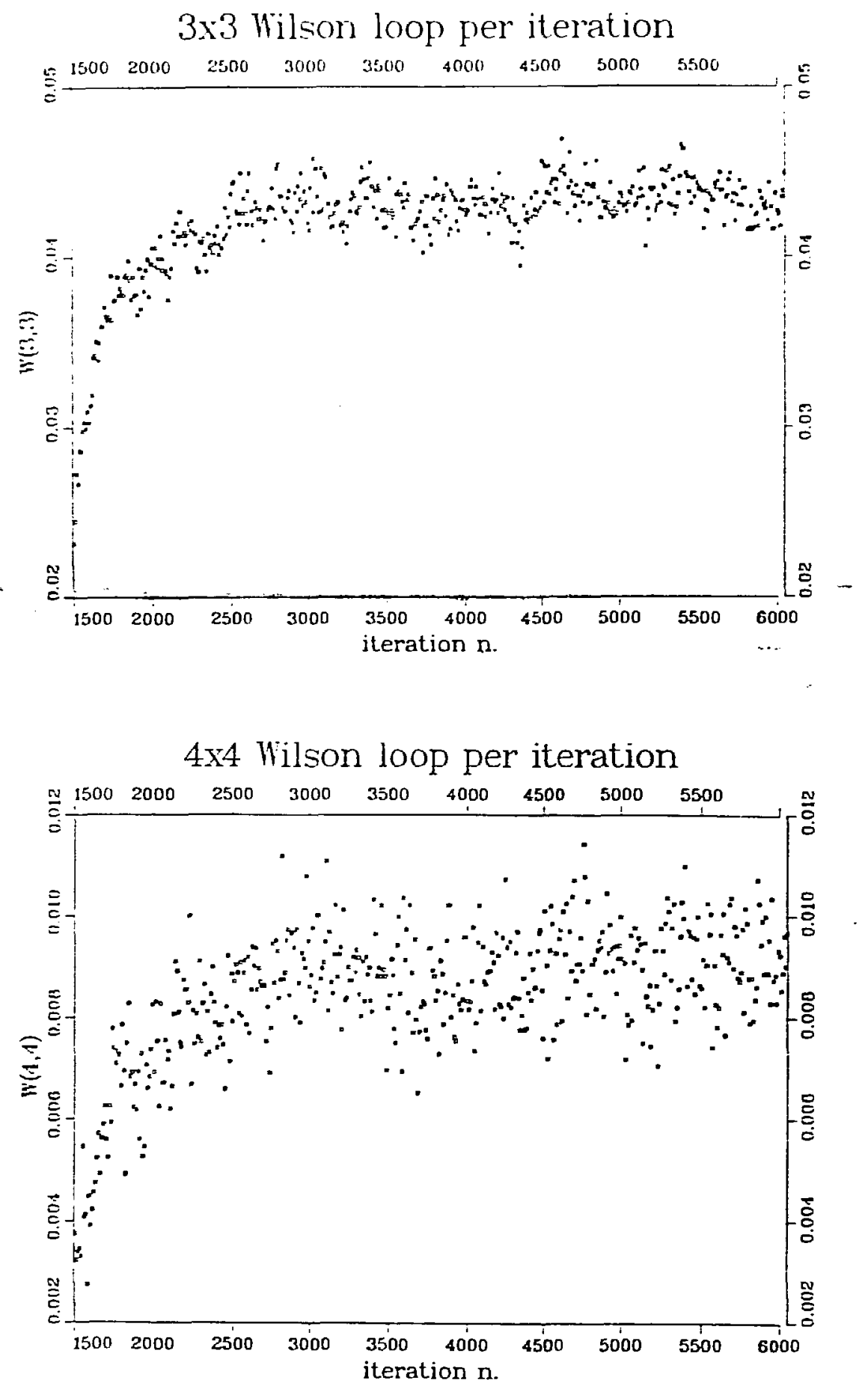

Fig. 2: $3 \times 3$ and $4 \times 4$ Wilson loops versus the iteration number. 


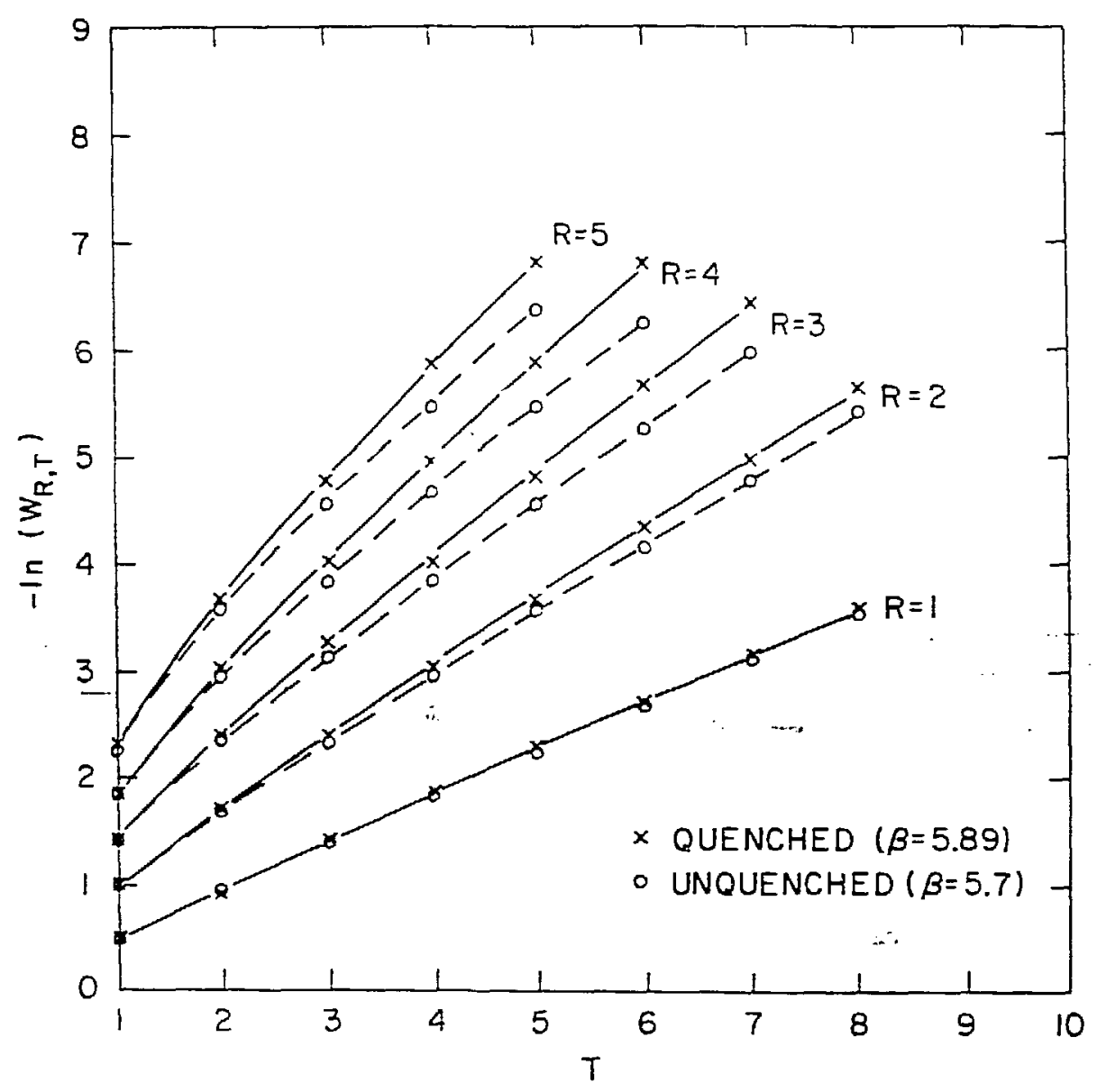

Fig. 3: Comparison of quenched and unquenched Wilson loops.

to be compared with the quenched value at the same coupling. ${ }^{8}$

$$
W(1,1)=0.5490 \pm .0010
$$

By running a quenched simulation on the same lattice, we have found that the value shown in (3) is reproduced within errors at $\beta=5.89$. Let us mention finally that a recent study by Gavai, Gocksch and Heller ${ }^{\circ}$ suggests that the pseudofermion algorithm may underestimate $W(1,1)$ at $m a=0.05$.

Figure 3 is a comparison of unquenched Wilson loops at $\beta=5.7$ and quenched ones at 5.89. It demonstrates that the effects of the dynamical fermions cannot be absorbed into a simple renormalization of $\beta, m$, or $n_{f}$. This confirms early claims by Gavai and $\mathrm{Karsch}^{10}$ ( $m a=0.10$ ). Let us point out however that on this issue Fukugita et $a .^{2}$ have concluded otnerwise. 


\section{HADRON PROPAGATORS}

The hadron propagators are deñned by ${ }^{3}$

$$
\begin{aligned}
\text { meson : } P_{t t_{0}}^{m(k)} & =\sum_{x} S_{x}^{k} \operatorname{tr} \Delta_{(x, t)}^{-1} \Delta_{\left(x, t_{0}\right)}^{-1} \\
\text { baryon : } P_{t t_{0}}^{h(t)} & =\sum_{x} S_{x}^{k} \operatorname{det} \Delta^{-1} \\
\Delta\left(x, t_{0}\right) & =(D+\tilde{m} a)_{x, t_{0}}
\end{aligned}
$$

The matrix $\Delta$ was inverted with the conjugate gradient algorithm in the following parameter range:

\begin{tabular}{|c|c|c|}
\hline$\tilde{m} a$ & C-G sweeps & residue (Barkai et al.[1]) \\
\hline \hline .50 & 50 & $10^{-8}$ \\
.10 & 100 & $10^{-7}$ \\
.05 & 200 & $10^{-7}$ \\
.02 & 350 & $10^{-6}$ \\
.01 & 500 & $10^{-6}$ \\
\hline
\end{tabular}

Figure 4 shows how the pion propagator $P_{t t_{0}}$ evolves with the (Metropolis) iteration number, for $\tilde{m} a=.05$ and $\tilde{m} a=.01$. Each continuous line $\therefore$ approximates the general path followed by the data as it migrates through the time slices, during a given iteration. This data is averaged over three source points ( $t_{0}$ ").

The nggure shows the propagator near $t=16$ to decrease by an order of magnitude over a range of at least 6000 iterations. Not surprisingly,the effect is more pronounced at $\tilde{m} a=.01$. In that case, 10000 are needed to get a good idea of how far down the minimum of $P_{t t_{0}}$ has to go. Not shown is the fact that it does go up again, from iteration 10000 to 14500 , without going beyond the 3000 line (this data was generated after this Lattice Meeting). In the case $\tilde{m} a=.05$, we need 6000 iterations to see the minimum go down and up again (fig. 4).

All this shows that one needs at least several thousands iterations to get a good idea of the drift of propagator values through the simulation. This is many more iterations than for $W(1,1)$. It is also many more than the 2000 commonly used in most previous quenched studies. We have verified this last point ourselves in our quenched runs at $\beta=5.89$ (fig. 5).

In the parameter range $m a=.05$ and $\tilde{m} a=.01, .05$, it appears that a pseudofermion calculation of the hadron mass spectrum with good 


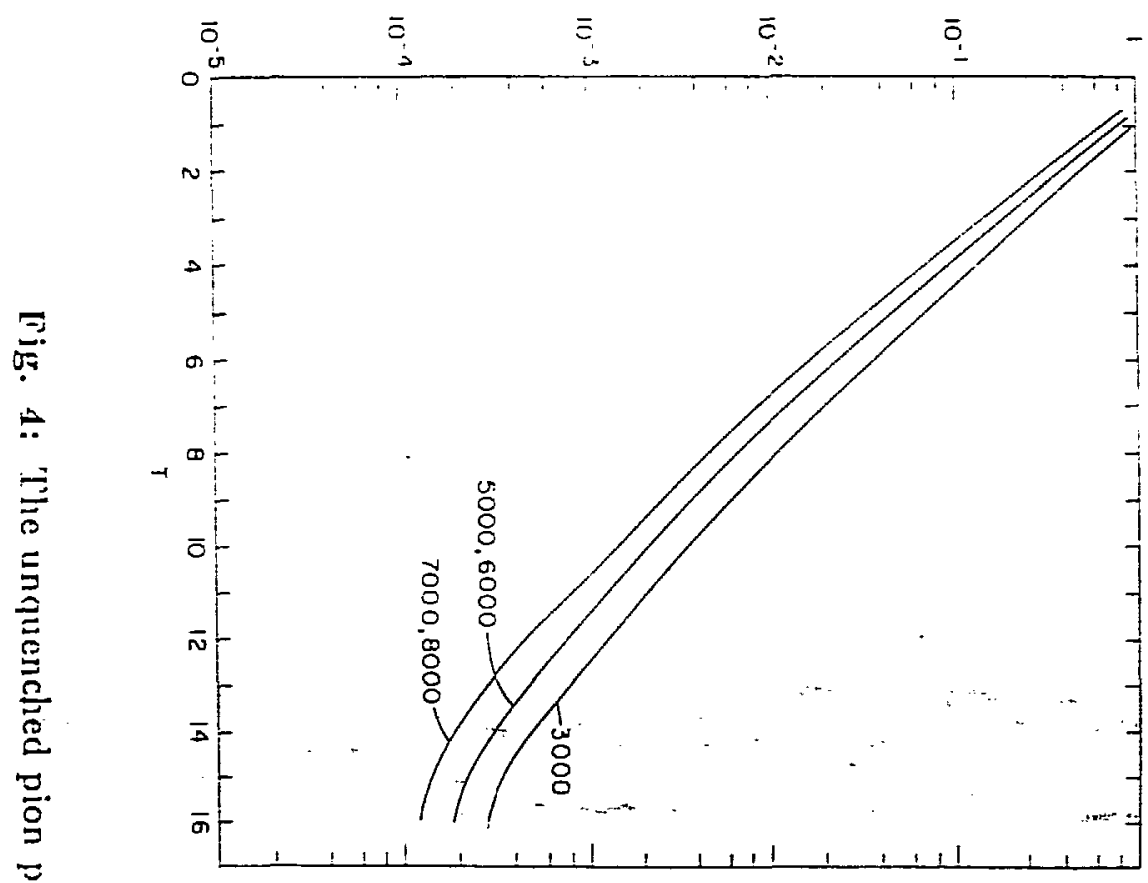

כ્ّ

$\widehat{E}$

تี

II

하

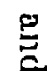

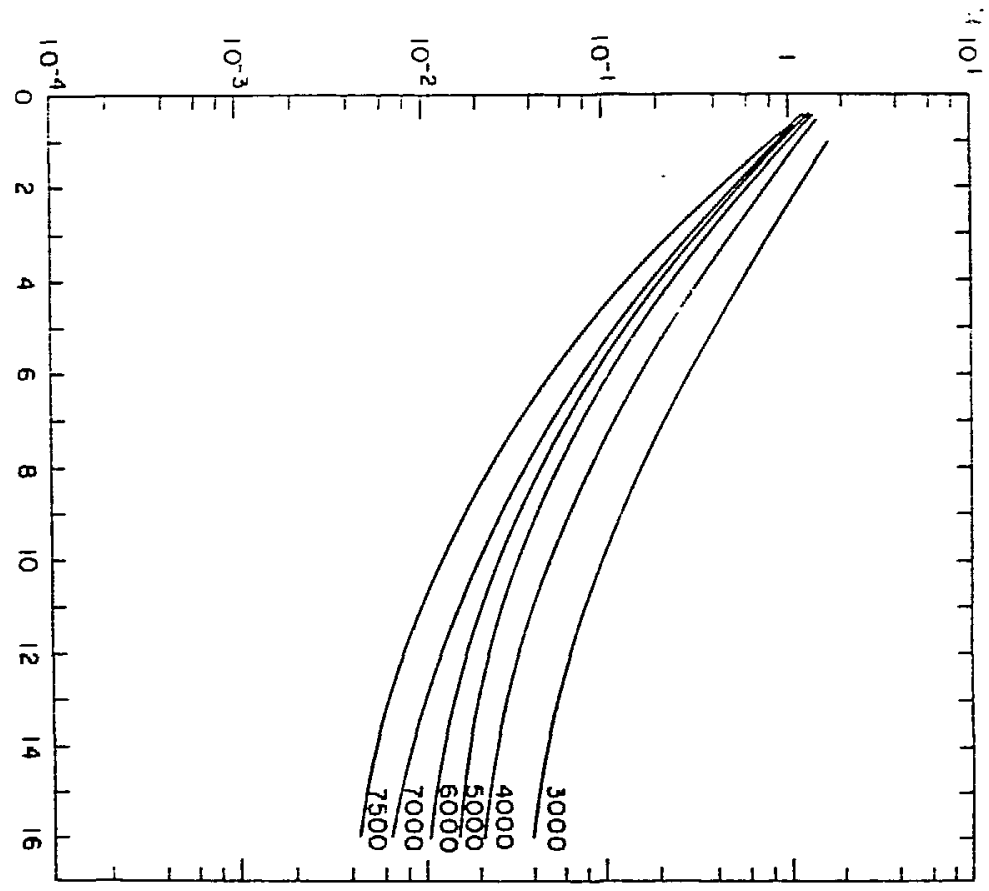



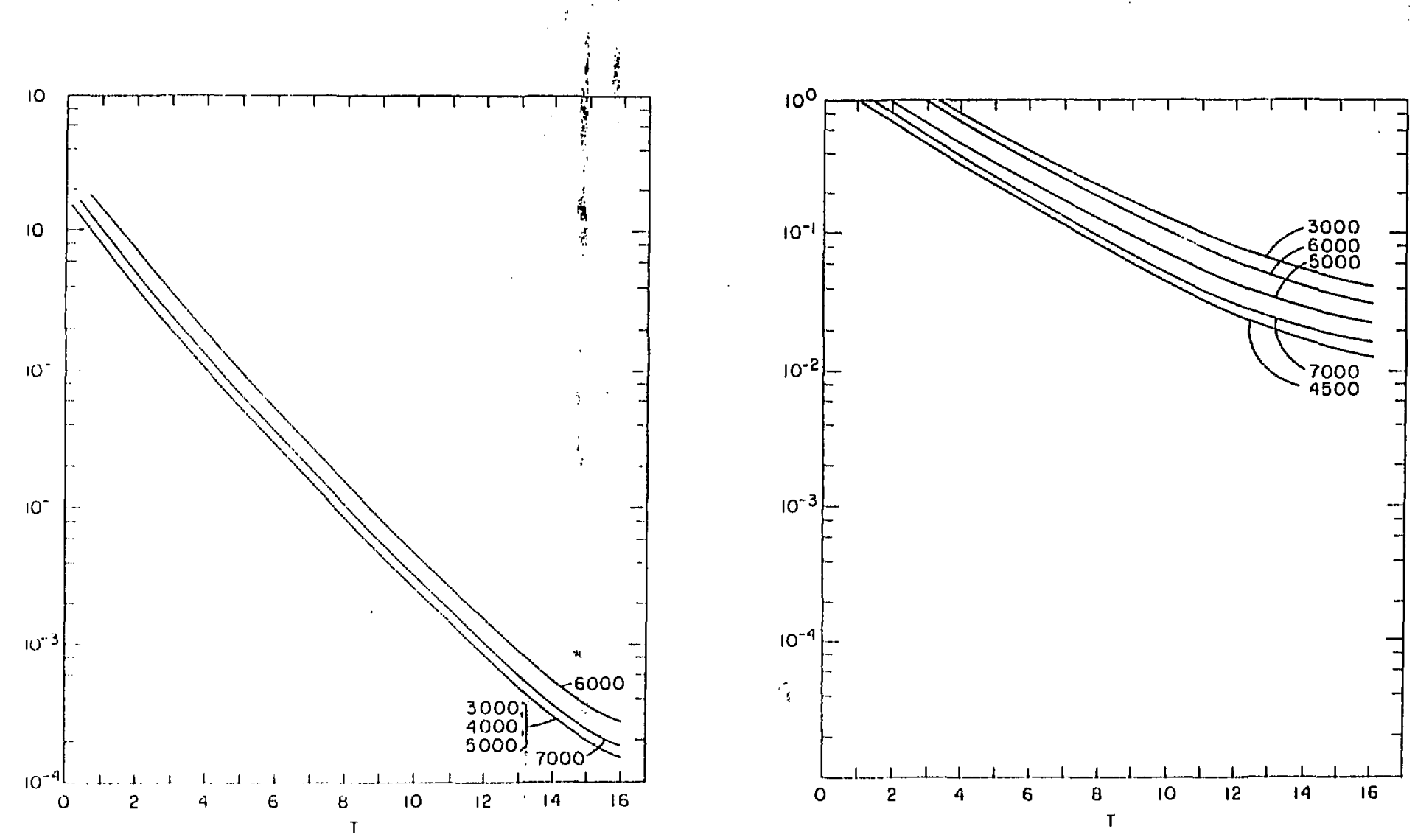

Fig. 5: The quenched pion propagator. (a) $\tilde{m} a=.05$ (b) $\tilde{m} a=.01$. 
statistics requires at least 4 to 5 times more iterations than in the quenched case. This is because a pseudofermion simulation has one more $(D+m a)$ inversion, which will also be affected by critical slowdown caused by small $m$.

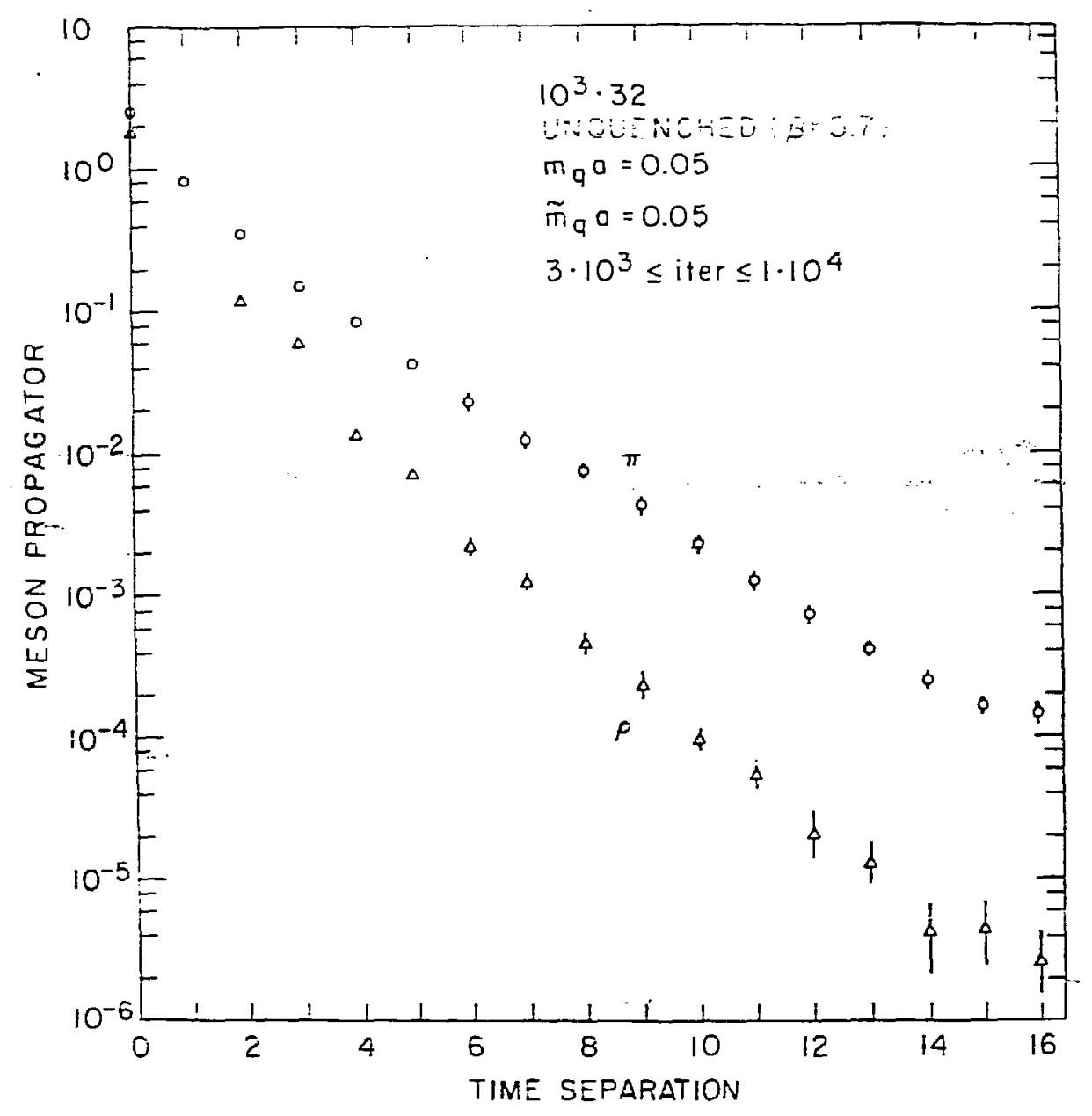

Fig. 6: The unquenched $\pi$ and $\rho$ propagators.

Figure 6 shows the resulting $\pi$ and $\rho$ unquenched propagators. The mass can be extracted according to standard methods; figure 7 displays preliminary pion masses, as function of $\sqrt{\tilde{\pi} a}$. The full analysis will be presented elsewhere. 


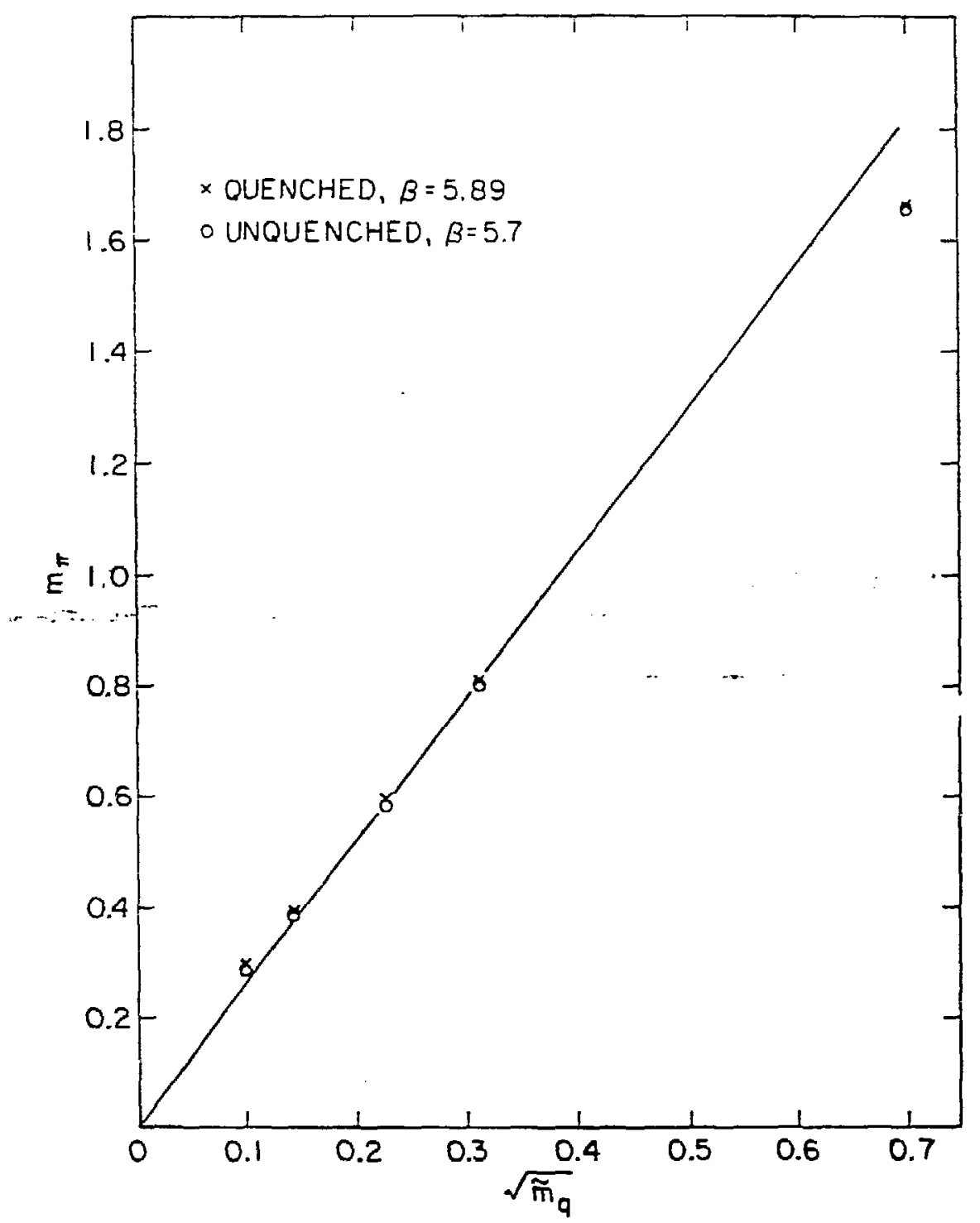

Fig. 7: Pion masses (unquenched).

\section{CONCLUSIONS}

To the best of our knowledge, this is the first demonstration of critical slow down of long wavelength structures in a background of dynamical fermions. Our propagators at $\tilde{m} a=m a=.05$ certainly need more than 8000 iteration on a $10^{3} \cdot 32$ lattice in order to get reasonably good statistics. In the case of $m a=.05 \tilde{m} a=.01$ the outlook is worse, as at least 10000 to 14500 are needed. This indicates how hard a similar study at $m a=\tilde{m} a=.01$ will 
be (we anticipate a factor 2 in iteration number). Compared with a quenched calculation, this represents 4 to 5 times more iterations, a hefty 40 to 50 -fold increase in computer time, if a factor of 0.1 is assumed for link upgrade in a quenched simulation.

This makes the importance of acceleration of long wavelength structures equilibration time all too obvious. Let us mention that tris question tion has already been addressed by the Cornell ${ }^{11}$ and the Kyoto-Tsukuba ${ }^{12}$ groups in the context of the Langevin equation.

The situation looks better in the case of Wilson loops, where 3000 or so iterations are required for equilibration. We have studied the $Q \bar{Q}-$ potential and found that the effects of dynamical fermions do not amount to a simple renorralization of the parameters.

\section{References}

1. For recent results see: K.C. Bowler, D.L. Chalmers, A. Kenway, R.D, Kenway, G.S. Pawley, D.J.Wallace, Nucl. Phys B240 [FS12] (1984) 213; J.P. Gilchrist, H.Schneider, G. Schierlhoiz and M. Teper Phys. Lett. 136B (1984) 87;

A. Billoire, E. Marinari and R. Petronzio, Nucl. Phys. B251 [FS13] (1985) 141 ;

D. Barkai, K.J.M. Moriarty and C. Rebbi, Phys. Lett. 156B (1985) 385;

A. Konig, K.H. Mütter, K. Schilling and J. Smit, Phys. Lett. 157B (1985) 421;

S. Itoh, Y. Iwasaki and T. Yoshie, Phys. Lett. 167B (1986) $443 .$.

2. M. Fukugita, Y. Oyanagi and A. Ukawa, Phys. Rev. Lett. 57 (1986) 953.

3. F. Fucito, K.J.M. Moriarty, C. Rebbi and S. Solomon, Phys. Lett. 172B (1986) 235.

4. E. Laermann, F. Langhammer, I. Schmitt and P. M. Zerwas, Phys. Lett. 173B (1986) 437 and 443.

5. F. Fucito, E. Marinari, G. Parisi and C. Rebbi, Nucl. Phys. B180 (1981) 360.

6. H.W. Hamber, E. Marinari, G. Parisi and C. Rebbi, Phys. Lett. 124B (1983) 99.

7. R.V. Gavai, Nucl. Phys. B269 (1986) 530.

8. A. Hasenfratz, P. Hasenfratz, U. Heller and F. Karsch, Phys. Lett. 143B (1984) 193;

M. Creutz and K.J.M. Moriarty, Phys.Rev. D26 (1982) 2166;

A. Hasenfratz, P. Hasenfratz, J. Heller and F. Karsch, Zeitschrift f. Ph. C25 (1984) 191.

9. R.V. Gavai, A. Gocksch and U. Heller, Nucl. Phys. (in press). 
10. R.V. Gavai and F. Karsch, Phys. Rev. Lett. 57 (1986) 40.

11. G.G. Batrouni, G.R. Katz, A.S. Fronfeld, G.P. Lepage, B. Svetitsky and K.G. Wilson, Phys. Rev. D32 (1985) 2736.

12. M. Fukugita, this conierence proceedings. 